\title{
Active Damping of a DC Network with a Constant Power Load: An Adaptive Observer-based Design
}

\author{
Juan E. Machado, Romeo Ortega, Alessandro Astolfi, \\ José Arocas-Pérez, Anton Pyrkin, Alexey Bobtsov, and Robert Griñó
}

\begin{abstract}
This paper proposes a nonlinear, output feedback, adaptive controller to increase the stability margin of a directcurrent (DC), small-scale, electrical network containing an unknown constant power load. Due to their negative incremental impedance, constant power loads are known to reduce the effective damping of a network, leading to voltage oscillations and even to network collapse. To overcome this drawback, we consider the incorporation of a controlled DC-DC power converter in parallel with the constant power load. The design of the control law for the converter is particularly challenging due to the existence of unmeasured states and unknown parameters. We propose a standard input-output linearization stage, to which a suitably tailored adaptive observer is added. The good performance of the controller is evaluated with numerical simulations.
\end{abstract}

\section{INTRODUCTION}

This note is concerned with the stability analysis of electrical networks with Constant Power Loads (CPLs). It is well-known that, due to their negative incremental impedance characteristic, CPLs induce voltage oscillations or even network collapse [1]. The analysis of networks with this type of loads started with [2], and has been an active research problem since then, see e.g., [3], [4] and [1]; we refer the reader to [5], and the references therein, for a recent review on this topic.

Various techniques have been explored for the stabilization of DC networks with CPLs-a survey may be found in [5]. These techniques are categorized into passive and active damping methods: the former are based on open-loop hardware alterations, whereas the latter imply the modification of existing —or added-control loops. In an active damping strategy, the control loops can be modified at three different network's positions [5]: at the source's side, at the load's side, and at a midpoint between them. In the present paper we are interested in the latter approach, which was firstly explored

Juan E. Machado and Romeo Ortega are with the Laboratoire des Signaux et Systèmes, CNRS-Supélec, Plateau du Moulon, 91192, France (emails: juan.machado@12s.centralesupelec.fr, romeo.ortega@1ss.supelec.fr).

A. Astolfi is with the Department of Electrical and Electronic Engineering, Imperial College London, London SW7 2AZ, UK and the DICII, Università di Roma "Tor Vergata", Via del Politecnico 1, 00133 Roma, Italy, Email: a.astolfi@imperial.ac.uk.

José Arocas-Pérez and Robert Griñó are with the Institute of Industrial and Control Engineering (IOC), Universitat Politècnica de Catalunya, 08028 Barcelona, Spain (e-mails: jose.arocas@upc.edu, roberto.grino@upc.edu).

Anton Pyrkin and Alexey Bobtsov are with the Department of Computer Science and Control Systems, ITMO University, Kronverkskiy av. 49, Saint Petersburg, 197101, Russia (e-mails: a.pyrkin@gmail.com, bobtsov@mail.ru). Anton Pyrkin is also with the Center for Technologies in Robotics and Mechatronics Components, Innopolis University, Innopolis, Russia. in [6], [7], and [8], for the stabilization of a small-scale network with a single CPL. In these references, the network's stabilization is achieved by adding a controlled power converter in parallel with the load and then, designing a suitable feedback control law for it: in [6], the converter is modeled as a simple controlled current source, and a linear control law is designed to stabilize the overall network; a similar approach, but using a full model for the power converter, is used in [7], however, their stabilization result is based on linearization of the network's dynamics; lastly, in [8] a large signal stability analysis, but using approximate techniques, such as the Takagi-Sugeno fuzzy model, is carried out to evaluate the performance of a linear controller.

The main contribution of this paper is described next. Following [6] and [7], we study the stabilization of a smallscale DC network supplying electrical energy to a CPL. First, we augment the network by placing a controlled power converter between the load and the source. Then, for the converter's control-loop design, instead of relying on linearfeedback techniques, we propose an adaptive observer-based nonlinear control law that provably achieves the overall network's stabilization. The control design is particularly challenging due to the existence of unmeasured statesthe current of the DC network-and the unknown power of the CPL. The construction of the proposed controller is based on the use of standard input-output linearization, to which a suitably tailored adaptive observer is added; its good performance is evaluated via numerical experiments. We point out that the stabilization problem addressed in this manuscript was previously studied in [9], where a statefeedback adaptive passivity-based control was proposed; the key differences between both works are specified in Remarks 2 and 3 of this note.

The rest of the paper is structured as follows. In Section II we present the model of the system under study and summarize its stability properties. The proposed controller configuration, adopted from [6] and [7], is presented in Section III. Our main stabilization results are included in Section IV. In Section V we present the numerical implementation of our theoretical developments. The note is finalized in Section VI with a number of concluding remarks and open problems that can be studied to extend our results.

\section{PROBlem Formulation}

\section{A. Description of the open-loop system}

The electrical network under study is shown in Fig. 1. It represents a simplified model of a DC power system and has 
been used in the literature, e.g., in [7], [10] and [11], to study the stability problems associated with CPLs. It is composed of a DC voltage source supplying energy to an instantaneous CPL. The transmission line is simply represented by the lossy inductor $L_{1}>0$ and the CPL is connected through the bus capacitor $C_{1}>0$. The network's dynamics are described by

$$
\begin{aligned}
L_{1} \dot{x}_{1} & =-r_{1} x_{1}-x_{2}+E, \\
C_{1} \dot{x}_{2} & =x_{1}-\frac{P}{x_{2}},
\end{aligned}
$$

where $x_{1}$ and $x_{2}$ denote the current through $L_{1}$ and the voltage across $C_{1}$, respectively. The constant parameter $P$ corresponds to the power extracted from, or injected to, the network by the CPL, being positive in the former case and negative in the latter-in the sequel, we focus our attention on the case $P \geq 0$. The state space for this system is defined as follows

$$
\mathcal{X}_{1}:=\left\{\left(x_{1}, x_{2}\right) \in \mathbb{R}^{2}: x_{2}>0\right\}
$$

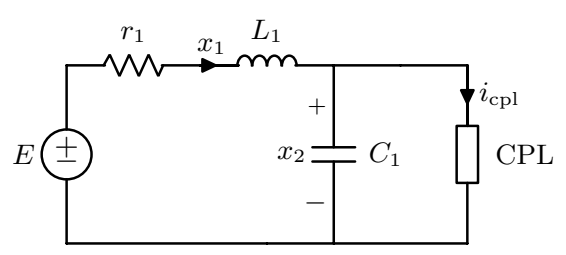

Fig. 1. A DC source supplying power to an instantaneous CPL.

\section{B. Equilibrium analysis of the open-loop system}

From [9], we enumerate the qualitative properties (QPs) of this network.

QP1 The system (1) has two real equilibria if and only if

$$
E^{2}-4 P r_{1} \geq 0 \Leftrightarrow P \leq \frac{E^{2}}{4 r_{1}} .
$$

QP2 One equilibrium corresponds to a high voltage/low current characteristic, which is stable only if

$$
P \leq \frac{E^{2} C_{1} L_{1} r_{1}}{\left(L_{1}+C_{1} r_{1}^{2}\right)^{2}},
$$

whenever $C_{1}<\frac{L_{1}}{r_{1}^{2}}$.

QP3 If $C_{1}>\frac{4 L_{1}}{r_{1}^{2}}$, the strict satisfaction of (2) is sufficient for asymptotic stability of the equilibrium mentioned in QP2.

Notice that if $P$ is negative, i.e., if the load behaves as a constant power source, then the expressions (2) and (3) are simultaneously satisfied; consequently, this scenario poses no treat regarding voltage collapse nor network's instability, hence our focus on the case $P \geq 0$.

\section{Objectives and methodology}

To introduce and support our methodology, we enlist the following remarks [9].

1) Observe from QP2 that if the capacitance $C_{1}$ is not big enough then, in order to maintain the system's stability, the power extraction from the CPL must be strictly smaller than the upper bound for existence of equilibria given in (2).

2) QP3 suggests a passive method to enlarge the domain of values of $P$ for which stability is ensured. It consists in increasing the effective capacitance $C_{1}$, which can be achieved with the open-loop parallel interconnection of a suitable capacitor and the CPL. Nonetheless, some disadvantages of this approach are reviewed in [6, Section III.A].

In view of these remarks, we specify our control objectives (COs) as follows.

CO1 Regulate the voltage $x_{2}$ around a constant value.

$\mathrm{CO} 2$ Relax the upper bound for $P$ established in (3).

CO3 Achieve these objectives without the knowledge of $P$.

As originally proposed in [6] and [7], to achieve these objectives we first add a power converter in parallel with the CPL, then we design a control strategy that stabilizes the overall network to a desired equilibrium point; the detailed description of the augmented circuit is carried out in the next section, and the presentation of the control law-which is the main contribution of the paper-is done in Section IV.

\section{Augmented Circuit Model}

We augment the network of Fig. 1 by adding a controlled DC-DC power converter in parallel with the load [6], [7], which results in the circuit shown in Fig. 2. The converter, which in the sequel is referred to as shunt damper, is composed of two complementary switches $u$ and $(1-u)$, a lossy inductor $L_{2}>0$, a capacitor $C_{2}>0$, and a resistor $r_{3}>0$; the latter models the losses associated with the switching devices.

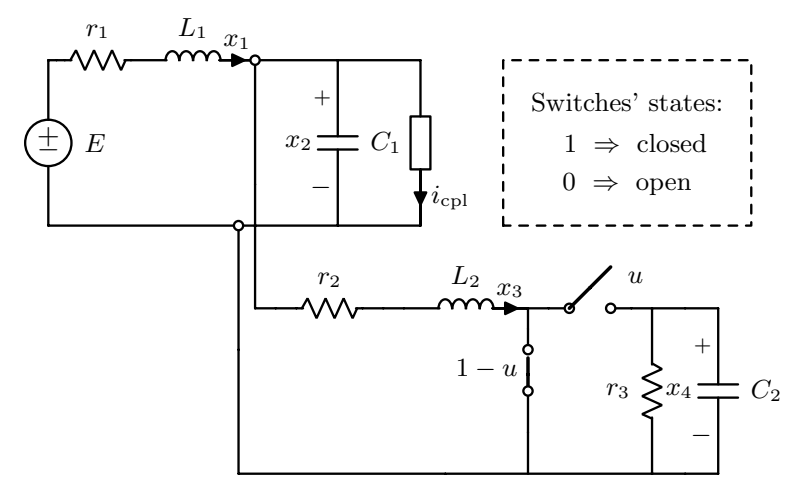

Fig. 2. The network of Fig. 1 is augmented by adding a power converter in parallel with the load.

The averaged dynamic model of the augmented system is given by

$$
\begin{aligned}
L_{1} \dot{x}_{1} & =-r_{1} x_{1}-x_{2}+E, \\
C_{1} \dot{x}_{2} & =x_{1}-\frac{P}{x_{2}}-x_{3}, \\
L_{2} \dot{x}_{3} & =-r_{2} x_{3}-u x_{4}+x_{2}, \\
C_{2} \dot{x}_{4} & =-\left(\frac{1}{r_{3}}\right) x_{4}+u x_{3},
\end{aligned}
$$


where $x_{3}$ is the current through $L_{2}, x_{4}$ is the voltage across $C_{2}$, and $u \in(0,1)$ - which is the system's control variablerepresents the duty cycle. The state space of the system is given as

$$
\mathcal{X}:=\left\{x \in \mathbb{R}^{4}: x_{2}>0, x_{4}>0\right\} .
$$

We conclude this section by making the important observations that $x_{1}$-being the current of a reduced model of the network-is not measurable, and the power $P$ is unknown.

\section{MAIN RESULTS}

In this section we propose a nonlinear, adaptive, outputfeedback controller that makes the augmented network (see Fig. 2) comply with the control objectives described in Subsection II-C. First, we analyze the equilibria of (4) and establish constraints, on the system's parameters, for their physical feasibility. Secondly, under the assumption that $x_{1}$ can be measured, and the constant parameter $P$ is known exactly, we present a full-information input-output linearization controller [12] that asymptotically stabilizes a specified equilibrium state. Finally, this controller is complemented with an observer for $x_{1}$ and an on-line estimator for $P$ yielding an adaptive, output-feedback controller design.

\section{A. Existence of equilibria}

A pair $(\bar{x}, \bar{u}) \in \mathcal{X} \times(0,1)$ is an equilibrium of (4) if and only if it belongs to the set ${ }^{1}$

$$
\mathcal{E}:=\{(\bar{x}, \bar{u}) \in \mathcal{X} \times \mathbb{R}: f(\bar{x})+g(\bar{x}) \bar{u}=0\} .
$$

An important issue in the design is to ensure that the power consumption of the shunt damper is minimal. In order to carry-out this analysis, we find convenient to parametrize $\bar{x}$ in terms of $\bar{u}$, as follows.

Proposition 1: Fix $0<\bar{u}<1$ as a desired steady state duty cycle of the shunt damper.

$\mathrm{C} 1(\bar{x}, \bar{u}) \in \mathcal{E}$ if and only if

$$
\Delta:=E^{2} \ell_{2}-4 P r_{1} \ell_{1} \geq 0,
$$

where we have defined

$$
\ell_{1}:=r_{3} \bar{u}^{2}+r_{1}+r_{2}, \ell_{2}:=r_{3} \bar{u}^{2}+r_{2}
$$

and

$$
\begin{aligned}
& \bar{x}_{1}=\frac{1}{2 r_{1} \ell_{1}}\left[E\left(\ell_{2}+2 r_{1}\right)-\sqrt{\ell_{2}} \sqrt{\Delta}\right], \\
& \bar{x}_{2}=\frac{1}{2 \ell_{1}}\left[\sqrt{\ell_{2}} \sqrt{\Delta}+E \ell_{2}\right], \\
& \bar{x}_{3}=\frac{1}{2 \ell_{1}}\left(\frac{\sqrt{\Delta}}{\sqrt{\ell_{2}}}+E\right), \\
& \bar{x}_{4}=\frac{r_{3} \bar{u}}{2 \ell_{1}}\left(\frac{\sqrt{\Delta}}{\sqrt{\ell_{2}}}+E\right) .
\end{aligned}
$$

\footnotetext{
${ }^{1}$ For space reasons we omit the explicit presentation of the obvious choice for the vector fields $f$ and $g$, that allow writing (4) as $\dot{x}=f(x)+g(x) u$.
}

C2 The power dissipated at the shunt damper in steady state, i.e., the quantity

$$
\begin{aligned}
\mathcal{P}_{\mathrm{L}} & :=r_{2} \bar{x}_{3}^{2}+\frac{1}{r_{3}} \bar{x}_{4}^{2}, \\
& =\frac{r_{3} \bar{u}}{4 \ell_{1}^{2}}\left(\frac{\sqrt{\Delta}}{\sqrt{\ell_{2}}}+E\right)^{2}
\end{aligned}
$$

attains a maximum at $P=0$ if and only if $\Delta>0$.

Remark 1: The condition $\Delta \geq 0$ in (5) is equivalent to the following upper bound for $P$,

$$
P \leq \frac{\ell_{2}}{\ell_{1}} \frac{E^{2}}{4 r_{1}}
$$

which can be proved to be a strictly increasing function of $\bar{u}$.

Remark 2: In [9], the equilibria $(\bar{x}, \bar{u}) \in \mathcal{E}$ are parametrized in terms of $\bar{x}_{2}$, not in terms of $\bar{u}$. Then, the equilibrium $\bar{x}$, associated to $\bar{x}_{2}=\frac{E}{2}$, is singled out for stabilization. This choice allows the network's stable operation in a wide range of values of $P$. Unfortunately, the steady-state shunt-damper's power dissipation is given in this case by $E^{2} / 4 r_{1}-P$, which implies a very low energetic efficiency when $P$ is small.

The parametrization that we propose in (6) implies a more involved algebraic expression for the damper's power losses, nonetheless, with an appropriate selection of $\bar{u}$ these losses can be made considerable inferior with respect to the latter approach.

This discussion is illustrated in Section V.

\section{B. Design of a full information stabilizing control law}

In this subsection we present a static, state-feedback, control law that renders asymptotically stable the equilibrium point (6). For its design, we assume that $P \geq 0$ is known, and fix $\bar{u} \in(0,1)$ such that (5) holds.

Following the ideas presented in [13, Section IV], we introduce the new input

$$
w=x_{4} u,
$$

which allows rewriting the system (4) in the cascade form shown in Fig 3, where

$$
\Sigma^{13}:\left\{\begin{array}{l}
L_{1} \dot{x}_{1}=-r_{1} x_{1}-x_{2}+E \\
C_{1} \dot{x}_{2}=x_{1}-\frac{P}{x_{2}}-x_{3} \\
L_{2} \dot{x}_{3}=-r_{2} x_{3}+x_{2}-w,
\end{array}\right.
$$

and

$$
\begin{gathered}
\Sigma^{4}: C_{2} \dot{x}_{4}=-\left(\frac{1}{r_{3}}\right) x_{4}+\frac{1}{x_{4}} w x_{3} . \\
\stackrel{w}{\longrightarrow \Sigma_{13}} \quad w x_{3} \Sigma_{4}
\end{gathered}
$$

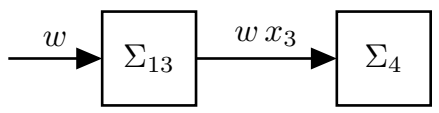

Fig. 3. Block diagram for the cascaded interconnection between the subsystems (7) and (8).

In the next proposition we present a control law that renders exponentially stable an equilibrium point of $\Sigma^{13}$. 
Proposition 2: Consider the system (7) and the output signal

$$
y:=x_{2}-\bar{x}_{2} \text {. }
$$

The static-state feedback

$$
\begin{aligned}
& w\left(x_{1}, x_{2}, x_{3}, P\right)=-L_{2} C_{1}\left[\beta\left(x_{2}-\bar{x}_{2}\right)+\alpha f_{2}\left(x_{1}, x_{2}, x_{3}\right)\right] \\
& +x_{2}-r_{2} x_{3}-L_{2}\left[f_{1}\left(x_{1}, x_{2}\right)+\frac{P}{x_{2}^{2}} f_{2}\left(x_{1}, x_{2}, x_{3}\right)\right]
\end{aligned}
$$

where

$$
\begin{aligned}
f_{1}\left(x_{1}, x_{2}\right) & :=\frac{1}{L_{1}}\left(r_{1} x_{1}-E+x_{2}\right), \\
f_{2}\left(x_{1}, x_{2}, x_{3}\right) & :=\frac{1}{C_{1}}\left(-x_{1}+\frac{P}{x_{2}}+x_{3}\right),
\end{aligned}
$$

is such that

$$
\ddot{y}+\alpha \dot{y}+\beta y=0 .
$$

Moreover, if $\alpha>0$ and $\beta>0$, the equilibrium point $\left(\bar{x}_{1}, \bar{x}_{2}, \bar{x}_{3}\right)$ is locally exponentially stable.

Next, we establish that if the subsystem $\Sigma^{13}$ is stable, then, $\Sigma^{4}$ is also stable.

Proposition 3: Let $\left(x_{1}, x_{2}, x_{3}\right)$ be any solution of the subsystem $\Sigma^{13}$ in closed-loop with the state-feedback (10) and starting sufficiently close to $\left(\bar{x}_{1}, \bar{x}_{2}, \bar{x}_{3}\right)$. Then, $x_{4}$, solution of $\Sigma^{4}$, is positive for all time and exponentially converges to $\bar{x}_{4}$, if it starts sufficiently close to it.

As a direct application of Propositions 2 and 3 we obtain the full-information input-output linearizing controller of the overall dynamics (4).

Proposition 4: Consider the system (4) in closed-loop with the static state-feedback control

$$
u=\frac{1}{x_{4}} w\left(x_{1}, x_{2}, x_{3}, P\right),
$$

where $w$ is given in (10). Then, $\bar{x} \in \mathcal{E}$ is a locally, exponentially stable equilibrium point of the closed-loop system.

\section{Stabilization assuming an unknown CPL power}

We now establish an adaptive version of the previously designed controller by adding an observer for $x_{1}$ and an online estimator for the constant parameter $P$, which are now assumed to be unmeasured and unknown, respectively.

Proposition 5: Consider the system (1) and assume that $x_{2}$ is positive and belongs to the interval $\left[x_{2}^{\min }, x_{2}^{\max }\right]$. Define the adaptive observer

$$
\begin{aligned}
\dot{q}_{1} & =\frac{E}{L_{1}}-\frac{x_{2}}{L_{1}}-\frac{r_{1}}{L_{1}} \hat{x}_{1}+k_{1} \hat{P}-k_{1} x_{2} \hat{x}_{1}+k_{1} x_{2} x_{3}, \\
\dot{q}_{2} & =-k_{2} \hat{P}+k_{2} x_{2} \hat{x}_{1}-k_{2} x_{2} x_{3}, \\
\hat{x}_{1} & =q_{1}+\frac{1}{2} k_{1} C_{1} x_{2}^{2}, \\
\hat{P} & =q_{2}-\frac{1}{2} k_{2} C_{1} x_{2}^{2},
\end{aligned}
$$

where $k_{1}$ and $k_{2}$ are such that

$$
\begin{aligned}
-\frac{r_{1}}{x_{2}^{\max } L_{1}} & <k_{1} \leq 0 \\
T_{1}\left(x_{2}^{\max }\right)-2 T_{2}\left(x_{2}^{\max }\right) & <k_{2}<T_{1}\left(x_{2}^{\max }\right)+2 T_{2}\left(x_{2}^{\max }\right),
\end{aligned}
$$

where

$$
\begin{aligned}
& T_{1}(\omega):=\frac{\omega k_{1} L_{1}+2 r_{1}}{\omega^{2} L_{1}} \\
& T_{2}(\omega):=\frac{1}{L_{1} \omega^{2}} \sqrt{\omega k_{1} L_{1} r_{1}+r_{1}^{2}} .
\end{aligned}
$$

Then $\left|\hat{x}_{1}-x_{1}\right| \rightarrow 0$ and $\hat{P} \rightarrow P$, exponentially fast.

The stability of (4) in closed-loop with an adaptive version of the control law (12) is established next.

Proposition 6: Let $k_{1}$ and $k_{2}$ be such that (14) hold. Fix $\bar{u}$ and compute $\bar{x}$ from (6). Define the adaptive control law

$$
u=\frac{1}{x_{4}} w\left(\hat{x}_{1}, x_{2}, x_{3}, \hat{P}\right),
$$

where $w$ is given in (10), and $\hat{x}_{1}, \hat{P}$ are generated by the adaptive observer (13). Then, $\left(x, \hat{x}_{1}, \hat{P}\right)=\left(\bar{x}, \bar{x}_{1}, P\right)$ is an asymptotically stable equilibrium point of the overall system.

Remark 3: A key extension with respect to the research done in [9] is the adaptive and simultaneous observation of the variable $x_{1}$ and the constant $P$. Its implementation requires the measurement of $x_{2}$ and $x_{3}$, which is easy to obtain in a physical setup.

Remark 4: The computation of the control law (12) requires the knowledge of $\bar{x}_{2}$, which is dependent on the unknown $P$; see equation (6). For the numerical results presented in the next section, $\bar{x}_{2}$ is computed from the estimate for $P$, i.e. $\hat{P}$, discretely, not continuously in time. This approach is common in hierarchical and supervisory control of AC and DC microgrids [14] and prevents introducing $\bar{x}_{2}$ to the controller if an overshoot of the time-varying signal $\hat{P}$ occurs, it also simplifies the controller design.

Remark 5: Through direct, but lengthy, computations, it can be shown that the condition (14) may be replaced by the conditions

$$
\begin{aligned}
0 & <k_{1}<\frac{8 r_{1}\left(x_{2}^{\min }+x_{2}^{\max }\right)}{L_{1}\left(x_{2}^{\max }-x_{2}^{\min }\right)^{2}}, \\
T_{1}\left(x_{2}^{\min }\right)-2 T_{2}\left(x_{2}^{\min }\right) & <k_{2}<T_{1}\left(x_{2}^{\max }\right)+2 T_{2}\left(x_{2}^{\max }\right) .
\end{aligned}
$$

\section{NUMERICAL VALIDATION}

In this section we present the results of two numerical experiments aimed at validating our theoretical findings. The physical parameters of the system are taken according to Table I, and the parameters for the adaptive controller of equation (15) are taken as

$$
\alpha=3 \times 10^{4}, \quad \beta=\frac{\alpha^{2}}{4}, \quad k_{1}=10, \quad k_{2}=4 \times 10^{5} .
$$

\section{A. Experiment No. 1}

We carried out the simulation of the closed-loop system (4) and (15). The equilibrium point to be stabilized, $\bar{x}$, is computed from equation (6), fixing $\bar{u}=0.5$. The initial condition has been taken as

$$
x(0)=\left.\bar{x}\right|_{P=0},
$$

which corresponds to an equilibrium point in the case $P=0$. Then, at $t=3 \mathrm{~s}$, a step change in the CPL power, from $P=0 \mathrm{~W}$ to $P=479 \mathrm{~W}$ has been introduced, this produces 


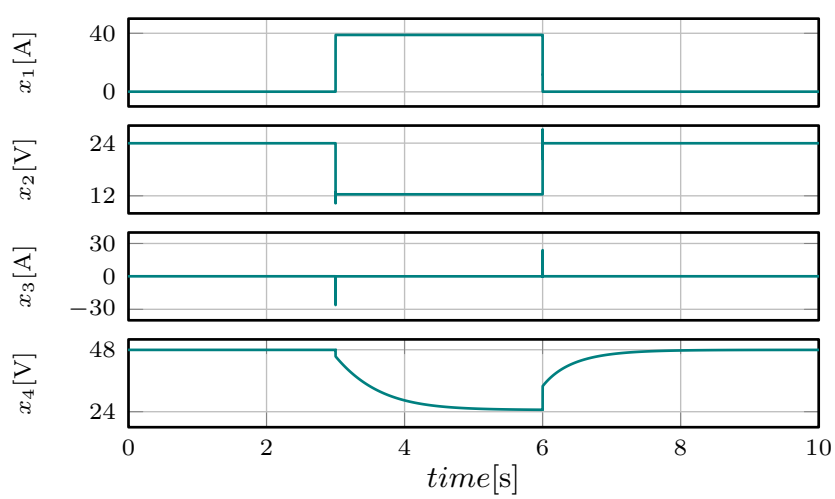

Fig. 4. Time history of $x$ for the experiment No. 1. Convergence is achieved despite the step change in the CPL's power.

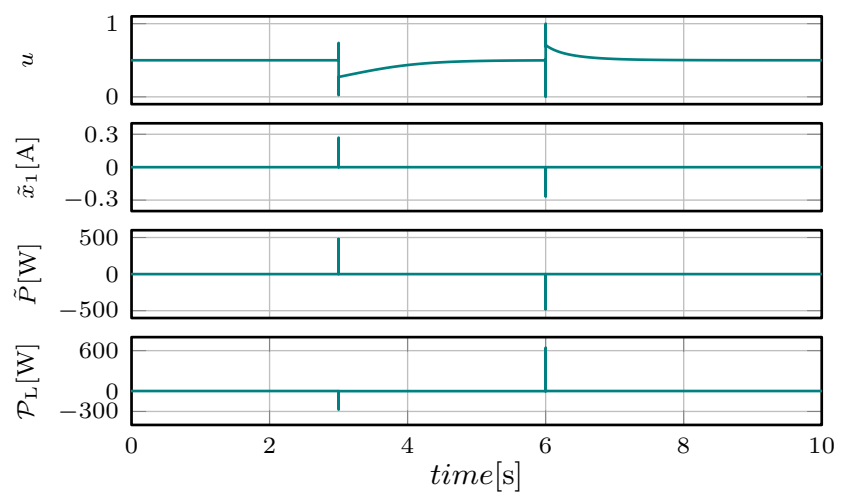

Fig. 5. Time histories of $u$, observation error for $x_{1}$ defined as $\tilde{x}_{1}=$ $x_{1}-\hat{x}_{1}$, power estimation error $\tilde{P}=P-\hat{P}$ and power loss $\mathcal{P}_{\mathrm{L}}=x_{2} x_{3}$ for the experiment No. 1 .

a new equilibrium point to be stabilized: $\left.\bar{x}\right|_{P=479}$. Lastly, at $t=6 \mathrm{~s}$, the CPL is stepped down to $0 \mathrm{~W}$ once again.

In Fig. 4 we show the time history of each component of the state variable $x$. The time histories of the control variable $u$, the error in the estimate of $x_{1}$, i.e., $\tilde{x}_{1}=x_{1}-\hat{x}_{1}$, the error $\tilde{P}=P-\hat{P}$, and the power consumption of the shunt damper, i.e., the product $\mathcal{P}_{\mathrm{L}}=x_{2} x_{3}$, are plotted in Fig. 5 .

\section{B. Experiment No. 2}

The setup is the same as for the previous experiment except that now we want to stabilize the equilibrium point $\bar{x}$ with $\bar{x}_{2}=\frac{E}{2}$ as done in [9]; see also Remark 2. The objective is to compare the power consumption of the power converter in both scenarios. The results are illustrated in Figures 6 and 7.

\section{Experiment's analysis}

Regarding the numerical experiments, the following remarks are in order.

1) $x$ converges to the desired equilibrium point in both cases.

2) $u \in(0,1)$ for all $t \geq 0$ in both experiments, making it physically feasible for the DC-DC converter. Nonetheless, a saturation function for $u$, to bound it between

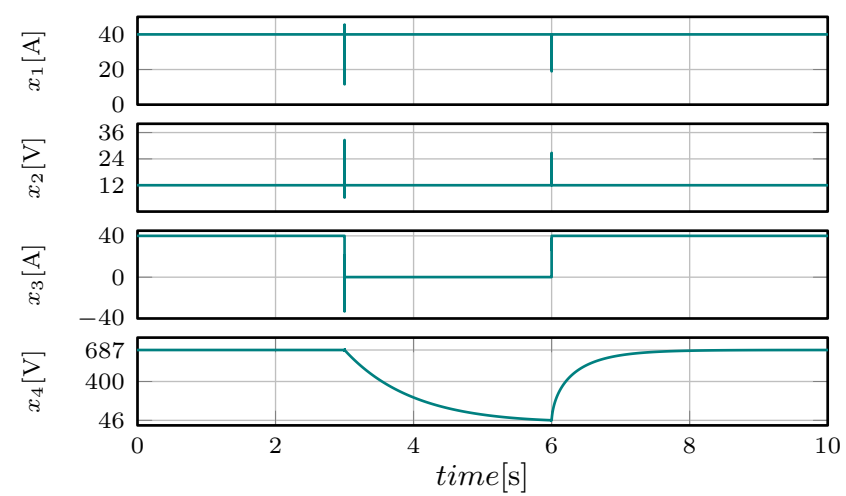

Fig. 6. Time history of $x$ for the experiment No. 2. Convergence is achieved despite the step change in the CPL's power.

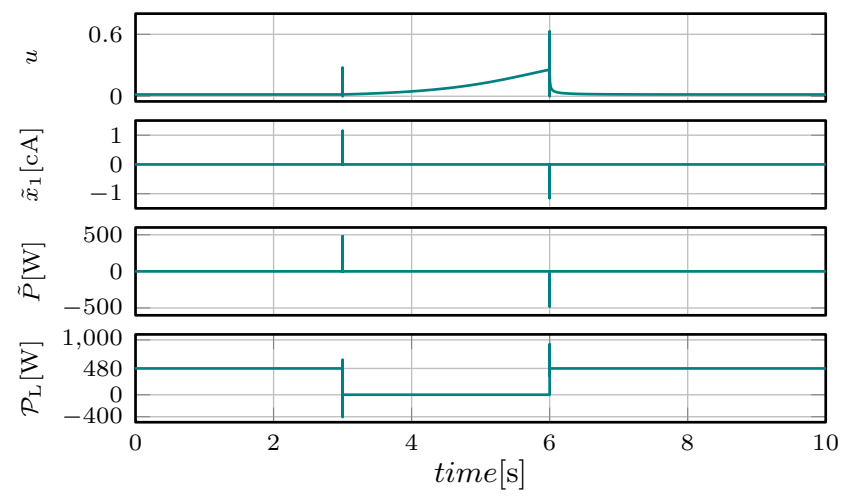

Fig. 7. Time histories of $u$, observation error for $x_{1}$ defined as $\tilde{x}_{1}=$ $x_{1}-\hat{x}_{1}$, power estimation error $\tilde{P}=P-\hat{P}$ and power loss $\mathcal{P}_{\mathrm{L}}=x_{2} x_{3}$ for the experiment No. 2.

zero and one, has been used; this prevents the control signal from overshooting.

3) The CPL changes its power discontinuously from $P=$ $0 \mathrm{~W}$ to $P=479 \mathrm{~W}$. This last value is well above the necessary bound for stability of the network without the shunt damper given in (3), which reads as

$$
\frac{E^{2} C_{1} L_{1} r_{1}}{\left(L_{1}+C_{1} r_{1}^{2}\right)^{2}}=276.9 \mathrm{~W} \text {. }
$$

Also, it satisfies the necessary and sufficient condition for existence of equilibria established in Subsection IIB .

4) The observer states $\hat{x}_{1}$ and $\hat{P}$ rapidly converge to $x_{1}$ and to $P$, respectively.

5) The power consumption of the shunt damper, $\mathcal{P}_{\mathrm{L}}$, is drastically higher for the experiment No. 2. Furthermore, in the same experiment, we can appreciate that the shunt damper output voltage, $x_{4}$, reaches prohibitively high values. In both senses, the controller's performance in the experiment No. 1 is superior.

\section{CONCLUSIONS AND FUTURE WORK}

In this paper we have proposed a nonlinear stabilization method for a DC small-scale power system supplying electric energy to a CPL. By adding a controlled DC-DC power 
TABLE I

PARAMETERS FOR THE CIRCUIT IN FIG. 2

\begin{tabular}{c|c|c|c}
\hline$r_{1}=0.3 \Omega$ & $L_{1}=85.0 \mu \mathrm{H}$ & $C_{1}=200 \mu \mathrm{F}$ & $E=24.0 \mathrm{~V}$ \\
\hline$r_{2}=5 m \Omega$ & $L_{2}=100 \mu \mathrm{H}$ & $C_{2}=1.0 \mathrm{mF}$ & $r_{3}=1 \mathrm{k} \Omega$ \\
\hline
\end{tabular}

converter in parallel with the load, we have been able to design, using standard input-output linearization, and a suitable tailored adaptive observer, a nonlinear adaptive control law for the stabilization of the overall network. Furthermore, our design permits the stable operation of the network for a wide range of values of the CPL and is able to relax some necessary stability bounds that are imposed if the system were to be operated in open-loop. Finally, satisfactory numerical experiments validate our theoretical results.

The results of this paper can be extended in the following directions.

- Explicitly compute estimates for the region of attraction of the equilibrium of the closed-loop system.

- Theoretically evaluate the robustness, against parameter uncertainty, of the proposed adaptive control.

- Extend our analysis to multi-port networks and with a distributed array of CPLs.

\section{ACKNOWLEDGMENTS}

Juan E. Machado was financially supported by the $\mathrm{Na}$ tional Council of Science and Technology (CONACyT for its acronym in Spanish) from Mexico. A. Astolfi has been partially supported by the European Union's Horizon 2020 Research and Innovation Programme under grant agreement No 739551 (KIOS CoE). José Arocas-Pérez and Robert Griñó were partially supported by the Government of Spain through the Agencia Estatal de Investigación Project DPI2017-85404-P and by the Generalitat de Catalunya through the Project 2017 SGR 872. A. Pyrkin and A. Bobtsov were partly supported by Government of Russian Federation (GOSZADANIE 2.8878.2017/8.9, grant 08-08), NSFC (61473183, U1509211).

\section{REFERENCES}

[1] A. Emadi, A. Khaligh, C. H. Rivetta, and G. A. Williamson, "Constant power loads and negative impedance instability in automotive systems: definition, modeling, stability, and control of power electronic converters and motor drives,' IEEE Transactions on Vehicular Technology, vol. 55, no. 4, pp. 1112-1125, 2006.

[2] R. D. Middlebrook, "Input filter considerations in design and application of switching regulators," IEEE Industry Applications Society Annual Meeting, pp. 366-382, 1976.

[3] M. Belkhayat, R. Cooley, and A. Witulski, "Large signal stability criteria for distributed systems with constant power loads," in IEEE Proceedings of the Power Electronics Specialist Conference (PESC'95), Atlanta, USA, 1995, pp. 1333-1338.

[4] M. Belkhayat, R. Cooley, and E. Abed, "Stability and dynamics of power systems with regulated converters," in IEEE Proceedings of ISCAS'95 - International Symposium on Circuits and Systems, Seattle, USA, 1995, pp. 143-145.

[5] S. Singh, A. R. Gautam, and D. Fulwani, "Constant power loads and their effects in dc distributed power systems: A review," Renewable and Sustainable Energy Reviews, vol. 72, pp. 407-421, 2017.

[6] M. S. Carmeli, D. Forlani, S. Grillo, R. Pinetti, E. Ragaini, and E. Tironi, "A stabilization method for dc networks with constantpower loads," in IEEE International Energy Conference and Exhibition (ENERGYCON), Florence, Italy, 2012, pp. 617-622.
[7] X. Zhang, X. Ruan, H. Kim, and K. T. Chi, "Adaptive active capacitor converter for improving stability of cascaded dc power supply system," IEEE Transactions on Power Electronics, vol. 28, no. 4, pp. 18071816, 2013.

[8] H. J. Kim, S. W. Kang, G. S. Seo, P. Jang, and B. H. Cho, "Largesignal stability analysis of dc power system with shunt active damper," IEEE Transactions on Industrial Electronics, vol. 63, no. 10, pp. 62706280, Oct 2016.

[9] J. E. Machado, J. Arocas-Pérez, W. He, R. Ortega, and R. Griñó, "Active damping of a dc network with a constant power load: An adaptive passivity-based control approach," in Congreso Nacional de Control Automático (CNCA), San Luis Potosi, Mexico, Oct 2018.

[10] H. Mosskull, "Optimal dc-link stabilization design," IEEE Transactions on Industrial Electronics, vol. 62, no. 8, pp. 5031-5044, Aug 2015.

[11] M. Wu and D. D. C. Lu, "A novel stabilization method of lc input filter with constant power loads without load performance compromise in dc microgrids," IEEE Transactions on Industrial Electronics, vol. 62, no. 7, pp. 4552-4562, July 2015.

[12] A. Isidori, Nonlinear control systems. Springer Science \& Business Media, 2013.

[13] R. Cisneros, F. Mancilla-David, and R. Ortega, "Passivity-based control of a grid-connected small-scale windmill with limited control authority," IEEE Journal of Emerging and Selected Topics in Power Electronics, vol. 1, no. 4, pp. 247-259, Dec 2013.

[14] J. M. Guerrero, J. C. Vasquez, J. Matas, L. G. De Vicuña, and M. Castilla, "Hierarchical control of droop-controlled ac and de microgrids - a general approach toward standardization," IEEE Transactions on industrial electronics, vol. 58, no. 1, pp. 158-172, 2011. 